\title{
Anti-Diabetic Effects of Edgeworthia chrysantha and Edgeworthia gardneri Flower Buds - An Ethnic Herbal Tea in China
}

\author{
Kit-Man Lau ${ }^{1,2}$, Chun-Wai Wong ${ }^{1,2}$, Chun-Lin Long ${ }^{3,4 *}$, Clara Bik-San Lau ${ }^{1,2 *}$ \\ ${ }^{1}$ Institute of Chinese Medicine, The Chinese University of Hong Kong, Shatin, New Territories, Hong Kong SAR, China \\ ${ }^{2}$ State Key Laboratory of Research on Bioactivities and Clinical Applications of Medicinal Plants, The Chinese University of Hong \\ Kong, Shatin, New Territories, Hong Kong SAR, China
}

${ }^{3}$ College of Life and Environmental Sciences, Minzu University of China, Beijing 100081, China

${ }^{4}$ Kunming Institute of Botany, Chinese Academy of Sciences, Kunming, Yunnan 650201, China

*Corresponding author: Clara Bik-San Lau, Institute of Chinese Medicine,The Chinese University of Hong Kong, Shatin, New

Territories, Hong Kong

Chun-Lin Long, College of Life and Environmental Sciences, Minzu University of China, China

\begin{abstract}
ARTICLE INFO
Received: 絊 June 29, 2020

Published: July 15, 2020

Citation: Kit-Man Lau, Chun-Wai Wong, Chun-Lin Long, Clara Bik-San Lau. Anti-Diabetic Effects of Edgeworthia chrysantha and Edgeworthia gardneri Flower Buds - An Ethnic Herbal Tea in China. Biomed J Sci \& Tech Res 28(5)-2020. BJSTR. MS.ID.004717.
\end{abstract}

Keywords: Edgeworthia chrysantha; Edgeworthia gardneri; EthnomedicinalPlant;EthnicGroups; Antihyperglycemic

Abbreviations: EC: Edgeworthia chrysantha Lindl; EG: Edgeworthia gardneri (Wall.) Meisn; HED: Human Equivalent Dose; OGTT: Oral Glucose Tolerance Test; STZ: Streptozotocin
ABSTRACT

Introduction: Edgeworthia chrysantha (EC) flower buds are consumed as tea by Tujia ethnic group in China. Owing to the close phytological origin and similar appearance, EC is believed to possess anti-diabetic effect like the related species Edgeworthia gardneri (EG).

Methods: This study aimed to test the hypothesis that EC aqueous extract was as potent as EG aqueous extract in lowering basal and postprandial plasma glucose levels of diabetic mice. The chemical profiles of the two extracts were also analyzed using ultra performance liquid chromatography.

Results: We found that EC aqueous extract contained $1.311 \%$ of tiliroside and $0.032 \%$ of daphnoretin, whereas EG aqueous extract contained $0.660 \%$ of tiliroside and $0.226 \%$ of daphnoretin. Neither edgeworin nor quercetin were detected in both extracts. Animal experiments demonstrated that EC and EG aqueous extracts at 200 $\mathrm{mg} / \mathrm{kg}$ (converted from human daily dose) were not potent in lowering basal plasma glucose levels of diabetic mice after 14-day treatment. However, a single oral dose of $200 \mathrm{mg} / \mathrm{kg}$ of EC or EG aqueous extract possessed mild antihyperglycemic effect in diabetic mice subjected to oral glucose tolerance test.

Conclusion: In conclusion, EC was of similar potency as EG and can be considered as an alternative of EG in diabetes management.

\section{Short Communication}

Edgeworthia chrysantha Lindl. (EC) of family Thymelaeaceae is a shrub widely distributed in east Asia. It is cultivated in Japan for making traditional Japanese paper (Washi) from its bark fibers. In China, this plant is mainly found in Henan Province, Shanxi Province and some other provinces along the southern side of Chang Jiang (the Yangtze River). According to Flora Reipublicae Popularis Sinicae, the whole plant of E. chrysantha is commonly used to heal bone fracture or rheumatalgia [1] as it possesses anti-inflammatory and 
analgesic activities [2]. In western Hunan Province, people of Tujia ethnic group pick the EC flower buds, dry them and then soak them in hot water to make herbal tea for daily consumption to tranquilize the mind, remove obstruction and improve digestion [3].

A closely related species Edgeworthia gardneri (Wall.) Meisn. (EG) distributes in areas at high altitude in eastern Tibet and northwest Yunnan Province. Its dried flower buds, named "Lu luohua" in Chinese, are marketed as precious Tibetan floral tea which is renowned for its broad pharmacological activities in treating diabetes mellitus, cardiovascular diseases and hyperlipidemia. As reported by Geng and colleagues, the water extract of EG flowers could inhibit $\beta$-D-glucosidase in vitro and significantly lower blood glucose level of alloxan-induced diabetic mice [4]. Recently, it was further elucidated that the water extract of EG flowers considerably decreased blood glucose level and ameliorated insulin resistance in diabetic mice through the regulation on gut bacterial phylotypes [5]. Owing to its promising anti-diabetic effect, EG has been used as the major component of a patented auxiliary blood sugardecreasing composite in China since 2014 [6].

Many compounds have been isolated from EC and EG flowers [7-10]. They can be mainly grouped as coumarins, flavonoids, flavone glycosides and triterpenes. Among these compounds, some isolated from EG have been proven to possess anti-diabetic effects. Edgeworin and daphnoretin were evaluated for their effects on two critical enzymes for carbohydrates digestion and metabolism. Edgeworin displayed strong inhibitory activity against $\alpha$-glucosidase, while daphnoretin exhibited potent inhibitory effect against both $\alpha$-glucosidase and $\alpha$-amylase [11]. Another compound, tiliroside,was identified as the major constituent of EG flowers. It showed a significant $\alpha$-glucosidase inhibitory activity and acted as a competitive inhibitor. Moreover, oral administration of tiliroside at $300 \mathrm{mg} / \mathrm{kg}$ could significantly reduce the fasting and postprandial blood glucose levels of streptozotocin (STZ)- induced diabetic mice [12]. Besides tiliroside, quercetin was also found to be a promising anti-diabetic agent. Quercetin induced insulin secretion in MIN-6 cell line in a glucose-dependent manner. Treatment of diabetic mice with quercetin resulted in remarkable decrease in blood glucose level, increase in insulin secretion and protection of pancreatic islets [13].

As shown in Figure 1, the appearance of flowers and dried flower buds of EC and EG are very similar in shape, color and size. Therefore, people of Tujia ethnic group very often collect EC flower buds as a substitute for EG and they believe that EC can help in managing diabetes mellitus as well. Although there are several scientific papers reporting the anti-diabetic effect of EG both in vitro and in vivo $[4,5,14,15]$, the effect of EC on basal or postprandial plasma glucose level is still unknown and needs to be investigated. Hence, in the present study, the hypoglycemic and antihyperglycemic effects of water extract of EC flower buds, in parallel with that of EG, were evaluated and compared in diabetic mice for the first time. Moreover, the chemical profiles of the two extracts were analyzed and the contents of those active anti-diabetic compounds (edgeworin, daphnoretin, tiliroside and quercetin) were quantified for comparison.

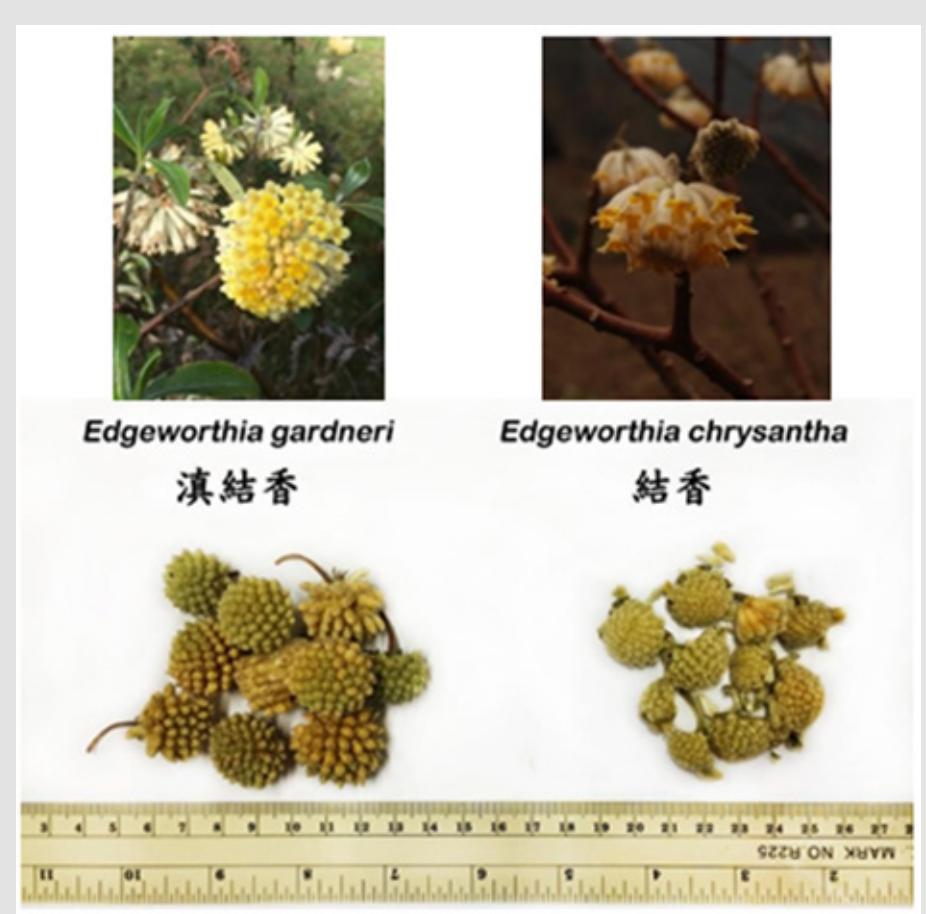

Figure 1: Flowers and dried flower buds of Edgeworthia gardneri (Wall.) Meisn. (EG) collected in Tengchong, Yunnan Province, China (Left) and Edgeworthia chrysantha Lindl. (EC) from Shaoyang, Hunan Province, China (Right). 


\section{Materials and Methods}

Dried flower buds of EC and EG were collected in Shaoyang of central Hunan and Tengchong of west Yunnan, respectively, and morphologically authenticated by Prof. C. L. Long. Herbarium voucher specimens were deposited at the museum of Institute of Chinese Medicine, The Chinese University of Hong Kong, with voucher specimen numbers assigned as 3483 for EC and 3494 for EG. Dried flower buds of EC or EG were extracted with distilled water under reflux twice. After lyophilization, the extraction yields for EC and EG were found to be $18.0 \%$ and $20.1 \%$, respectively.

UPLC analyses of aqueous extracts of EC and EG were conducted using Waters UPLC system (MA, USA). The column used was Agilent ZORBAX Eclipse Plus C18 RRHD, 3.0 x 100 mm, $1.8 \mu \mathrm{m}$. The chromatographic separation was conducted at $40^{\circ} \mathrm{C}$ under gradient conditions at a flow rate of $0.5 \mathrm{~mL} / \mathrm{min}$. The $\mathrm{LC}$ profile was as follows: Mobile phase: (A) $0.1 \%$ formic acid in deionized water and (B) acetonitrile; Gradient: 0-1 min, 5 \% B; 1-10 min, 5-21 \% B; 10-15 min, 21 \% B; 15-19 min, 21-25\% B; 19-25 min, $25 \%$ B; 25-36 min, 25-47 \% B. The column was flushed with $100 \%$ B for 3 min and re-equilibrated for another $3 \mathrm{~min}$ after each injection. UV 249 $\mathrm{nm}, 314 \mathrm{~nm}, 343 \mathrm{~nm}$ and $371 \mathrm{~nm}$ were used to detect edgeworin, tiliroside, daphnoretin and quercetin, respectively.

Diabetic $(+d b /+d b)$ mice $(5.5-7$ weeks old, male) were supplied by Laboratory Animal Service Centre, The Chinese University of Hong Kong. They were housed under the conditions of $22-25^{\circ} \mathrm{C}$ and a 12 -hour light-dark cycle. Standard animal chow and tap water were accessed ad libitum. Hypoglycemic effect and antihyperglycemic effect in Oral Glucose Tolerance Test (OGTT) of aqueous extracts of EC and EG on overnight-fasted diabetic mice were performed as previously reported with minor modifications [16]. Metformin (200 mg/kg) was used as a positive control.

In general, one person consumes $5 \mathrm{~g}$ of dried flower buds a day. As the aforementioned extraction yield is about $20 \%$, one person intakes $1 \mathrm{~g}$ of extract powder daily. Human Equivalent Dose (HED) is $1 \mathrm{~g} / 60 \mathrm{~kg}$, i.e. $16.7 \mathrm{mg} / \mathrm{kg}$. After normalization of body surface area [17], mouse dose is 16.7 x 37/3,i.e. $206 \mathrm{mg} / \mathrm{kg}$. In the animal studies, $200 \mathrm{mg} / \mathrm{kg}$ was therefore used as the testing dose for both $\mathrm{EC}$ and $\mathrm{EG}$ aqueous extracts.

\section{Results}

The UPLC profiles of EC and EG aqueous extracts are shown in Figure 2. It was found that the active compounds edgeworin and quercetin were not present in the extracts. On the other hand, tiliroside and daphnoretin could be detected. Their amounts were quantified and listed in Table 1. Effects of 14-day treatment with EC or EG aqueous extract on body weights and basal plasma glucose levels of overnight-fasted diabetic mice are shown in Figure 3. It was found that the body weights of all groups of mice increased gradually during the course of experiment (Figure 3A). However, weight gain of mice in EG group was the least. On average, their weight increased by $7.7 \%$ on Day 15 when compared with that on Day 1 whilst other groups, including water, EC and metformin groups, had around $17 \%$ increase in body weight. As shown in Figure $3 \mathrm{~B}$, the mice of EC and EG groups had lower plasma glucose levels than the water (control) group on Day 8. However, the effect was not statistically significant and the trend of decrease disappeared on Day 15. It implied that EC or EG aqueous extract at $200 \mathrm{mg} / \mathrm{kg}$ did not exert hypoglycemic effect in our current experiment setting.

Table 1: Amounts of edgeworin, tiliroside, daphnoretin and quercetin in EC or EG aqueous extracts. Data are mean $\pm \mathrm{SD}$ $(n=3)$.

\begin{tabular}{|c|c|c|}
\hline \multirow{2}{*}{} & $\begin{array}{c}\text { Amount in EC } \\
\text { aqueous extract }\end{array}$ & $\begin{array}{c}\text { Amount in EG } \\
\text { aqueous extract }\end{array}$ \\
\cline { 2 - 3 } & $\mathbf{( \% , w / w )}$ & $\mathbf{( \% , w / w )}$ \\
\hline Edgeworin & $<0.0001$ & $<0.0001$ \\
\hline Tiliroside & $1.311 \pm 0.052$ & $0.660 \pm 0.029$ \\
\hline Daphnoretin & $0.032 \pm 0.001$ & $0.226 \pm 0.009$ \\
\hline Quercetin & $<0.0002$ & $<0.0002$ \\
\hline
\end{tabular}

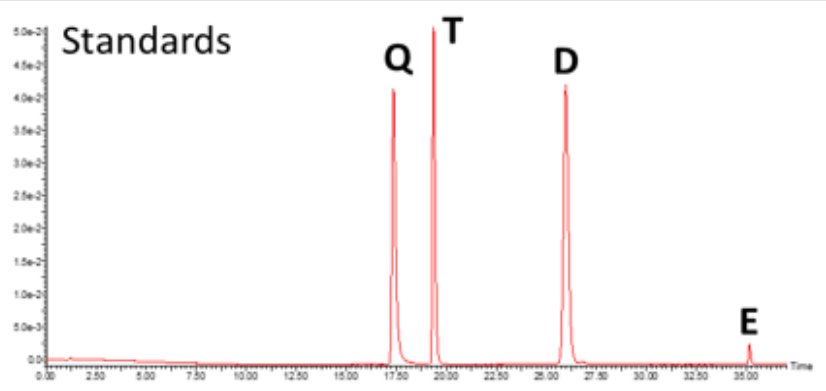

EG
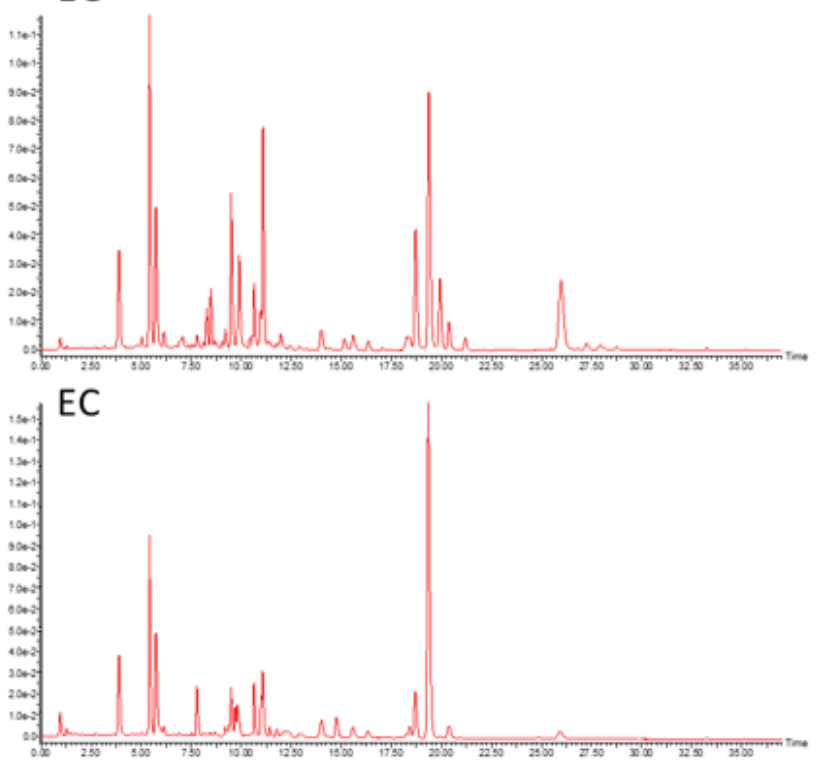

Figure 2: UPLC profiles of standards mixture, EG aqueous extract and EC aqueous extract at UV $343 \mathrm{~nm}$. Q: quercetin; T: tiliroside; D: daphnoretin; E: edgeworin. 

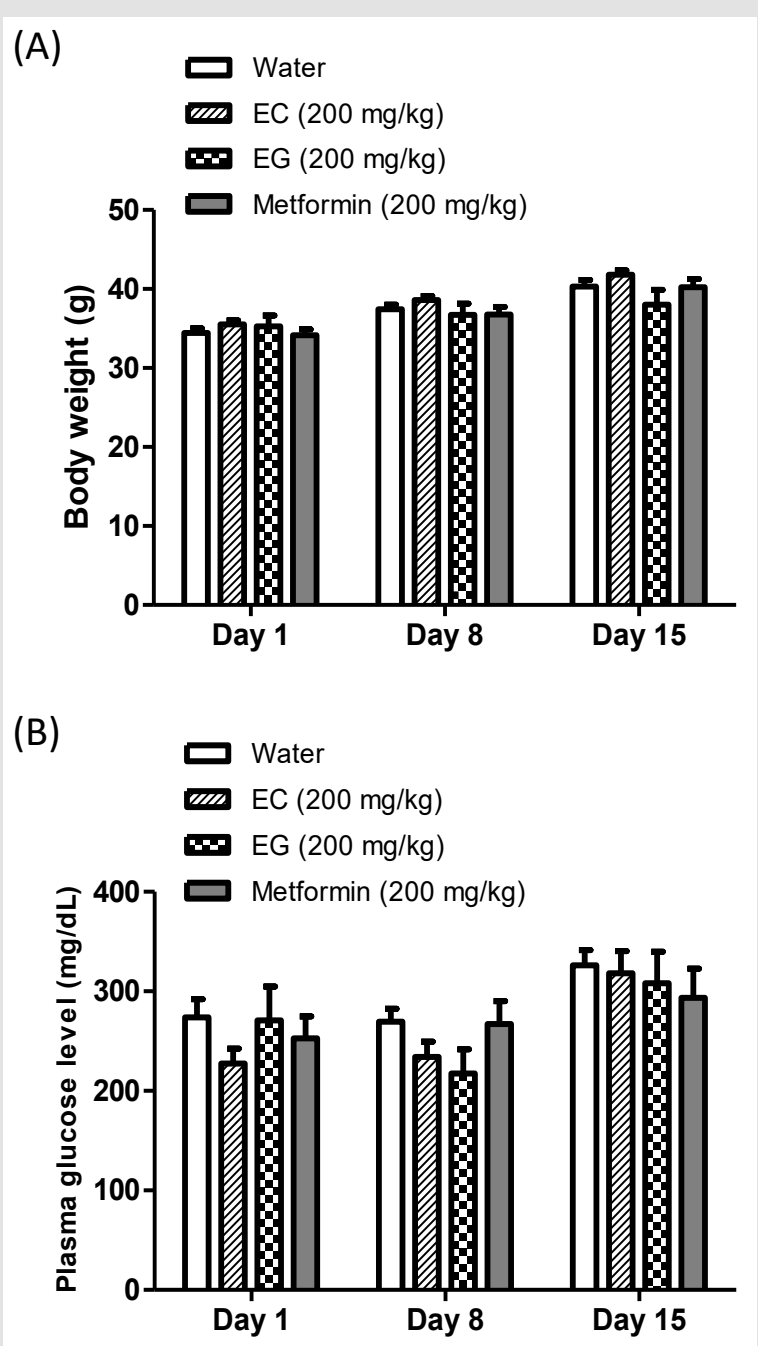

Figure 3:

(A) Body weights and

(B) Basal plasma glucose levels of overnight-fasted diabetic mice during the course of 14-day treatment with EC or EG aqueous extract at $200 \mathrm{mg} / \mathrm{kg}$.

Data are mean \pm SEM $(n=6-13)$. No significant difference was observed among groups on the same experiment day.

Results of OGTT are shown in Figure 4. As the positive control, metformin could significantly lower plasma glucose levels of diabetic mice after glucose challenge when compared with that of water (control) group ( $\mathrm{p}<0.05)$. After a single treatment of $200 \mathrm{mg}$ / $\mathrm{kg}$ of EC or EG aqueous extract, a trend of antihyperglycemic effect was observed. However, no statistical significance was detected (Figure 4). 


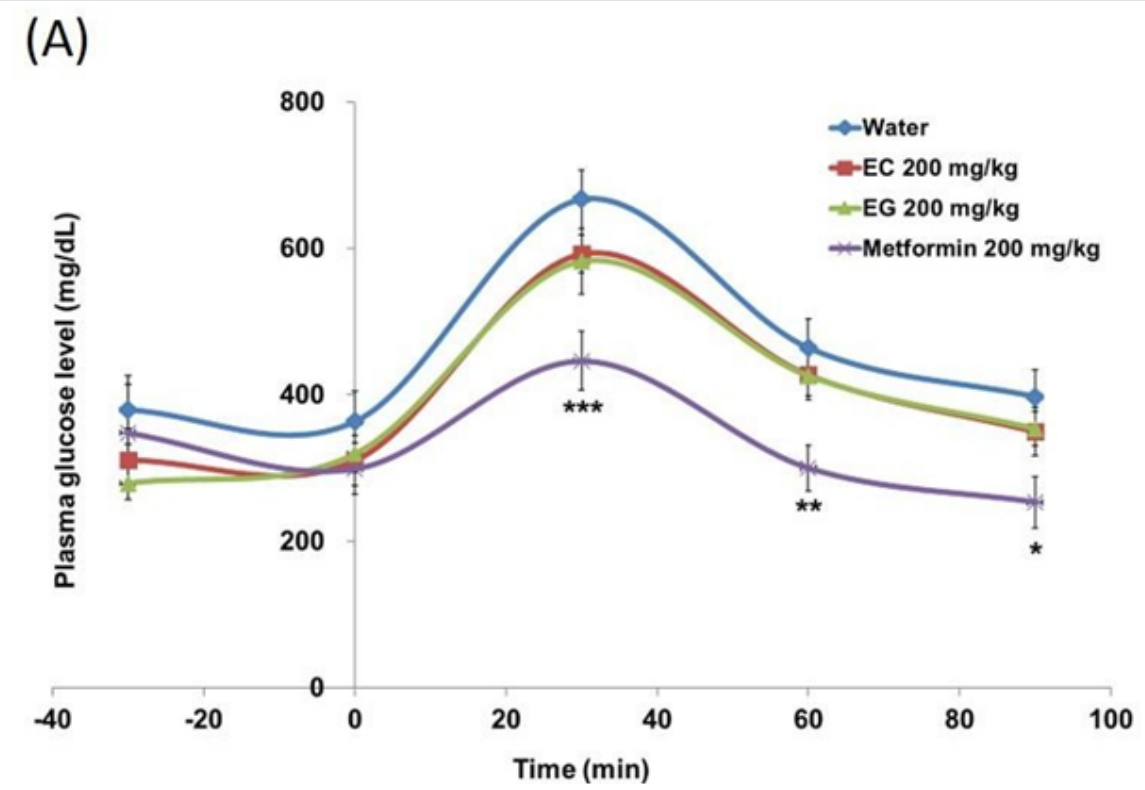

(B)

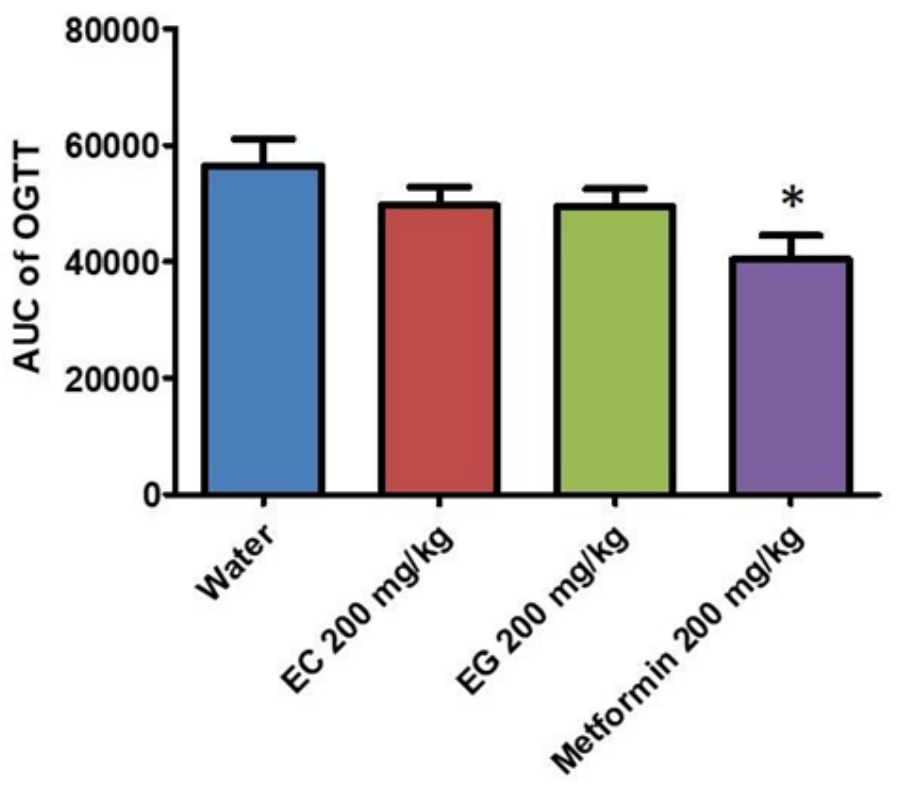

Figure 4: Effect of EC and EG aqueous extracts on Oral Glucose Tolerance Test (OGTT) in diabetic mice. (A) Plasma glucose levels of different groups at different time points. Plasma samples were taken before drug treatment (-30 min), before glucose challenge ( $0 \mathrm{~min}$ ), and various time points after glucose challenge (30 to $90 \mathrm{~min}$ ). (B) Area under the curve (AUC) of each curve plotted in Figure 4(A). Data are mean \pm SEM $(n=9-14) .{ }^{*} p<0.05,{ }^{* *} p<0.01$ and ${ }^{* * *} p<0.001$ when compared with water (control) group.

\section{Discussion}

As a substitute of EG, flower buds of EC are always used by people of Tujia ethnic group for the management of diabetes mellitus. In the present study, the chemical composition and anti-diabetic effect of EC aqueous extract were investigated and compared with that of EG.
In the hypoglycemic animal experiment, the weight gain of mice in EG group was the least (Figure 3A). Moreover, 4 out of 10 mice in EG group died before the end of experiment (data not shown). Taking these pieces of information together, the dosage of EG at $200 \mathrm{mg} /$ $\mathrm{kg}$ might be too high for diabetic $(+\mathrm{db} /+\mathrm{db})$ mice. However, Geng 
and colleagues reported that single intragastric administration of EG aqueous extract at 1000, 2500 and $5000 \mathrm{mg} / \mathrm{kg}$ did not cause any acute toxicity to normal mice [4]. The inconsistency between our findings and the reported ones might be due to the variations in animal strains, animal conditions and dosing intervention. On the other hand, continuous treatment with EC at $200 \mathrm{mg} / \mathrm{kg}$ for 14 days seemed to be safe to the animals.

It was reported that intragastric application of aqueous extract of EG at 1000, 2000 and $3000 \mathrm{mg} / \mathrm{kg}$ for 4 weeks could lower fasting blood glucose of high-fat diet- and STZ-induced diabetic mice [18]. However, the hypoglycemic effect of EG at lower dosage (200 mg/kg) for shorter treatment duration (14 days) could not be demonstrated in our study using genetically diabetic mice. Similar to EG, EC did not exhibit any promising effect in lowering basal blood glucose level either (Figure 3B).

According to traditional practice, floral tea is usually prepared by soaking flower buds in hot water. Under this extraction condition, edgeworin and quercetin are not available for consumption as reflected by the fact that they were not detected in EC or EG aqueous extract (Table 1). Even though they have been reported to possess anti-diabetic effects[11,13], edgeworin and quercetin were unlikely to contribute to the activity of EC or EG aqueous extract.

Tiliroside was identified as the major constituent of EG flowers [12]. As determined using UPLC, EC aquoues extract contained $1.311 \%$ of tiliroside, which was double the amount in EG aqueous extract $(0.660 \%)$ (Table 1$)$. It has been reported that tiliroside at a dose of $300 \mathrm{mg} / \mathrm{kg}$ could significantly reduce the fasting and postprandial blood glucose levels of STZ-induced diabetic mice [12]. However, tiliroside might not have contributed a lot to the antihyperglycemic effect of EC or EG (Figure 4) because its actual amount in $200 \mathrm{mg} / \mathrm{kg}$ of EC and EG aqueous extracts were only 2.6 $\mathrm{mg} / \mathrm{kg}$ and $1.3 \mathrm{mg} / \mathrm{kg}$, respectively, which were much lower than the reported active dose $(300 \mathrm{mg} / \mathrm{kg})$. Therefore, the in vivo antidiabetic effect of EC aqueous extract was not significantly different from that of EG aqueous extract, despite containing higher tiliroside content.

\section{Conclusion}

In conclusion, the results of the present study showed for the first time the mild antihyperglycemic effect of EC, supporting its traditional usage by ethnic people in China. At tested dosage (200 $\mathrm{mg} / \mathrm{kg}$, according to traditional human use in making tea), EC and EG exhibited similar potency. Although EG is more common and commercially available as products, our findings suggested that EC can also be considered as an alternative of EG in terms of diabetes management.

\section{Acknowledgement}

The authors would like to thank Mr. Ching-Po Lau of Institute of Chinese Medicine, The Chinese University of Hong Kong for his help in preparing the aqueous extracts.

\section{Statement of Ethics}

Procedures adopted in studies involving animals have been approved by Animal Experimentation Ethics Committee (AEEC), The Chinese University of Hong Kong (AEEC Approval No.: 15-165MIS).

\section{Conflict of interest}

The authors have no conflicts of interest to declare.

\section{Funding Sources}

This work was financially supported by grants of the State Key Laboratory of Research on Bioactivities and Clinical Applications of Medicinal Plants (CUHK) from HKSAR, the National Natural Science Foundation of China (31761143001, 31870316), Key Laboratory of Ethnomedicine (Minzu University of China) of Ministry of Education of China (KLEM-ZZ201904, KLEM-ZZ201906), Ministry of Ecology and Environment (2019HB2096001006), and Ministry of Education of China and State Administration of Foreign Experts Affairs of China (B08044).

\section{References}

1. (1999) Editorial Committee of Flora Reipublicae Popularis Sinicae of the Chinese Academy of Sciences. Flora Reipublicae Popularis Sinicae. Beijing: Science Press 52(1); 391-392. (in Chinese)

2. $\mathrm{Hu} X J$, Jin $\mathrm{HZ}, \mathrm{Xu}$ WZ, Chen M, Liu XH, et al. (2008) Anti-inflammatory and analgesic activities of Edgeworthia chrysantha and its effective chemical constituents. Biol Pharm Bull 31(9): 1761-1765.

3. Yang DS, Li LH, Tian XR, Yang DQ (2016) Practical Medicine of Tujiazu. $1^{\text {st }}$ edn Beijing: China Medical Science Press p. 436. (in Chinese)

4. Geng Y, Yang H, Xu H, Shi J (2013) $\alpha$-Glucosidase inhibitory activity of the alabastrum of Edgeworthia gardneri (Wall.) Meisn. J Food Sci Biotechnol 32(9): 967-971. (in Chinese)

5. Zhang Z, Xu H, Zhao H, Geng Y, Ren Y, et al. (2019) Edgeworthia gardneri (Wall.) Meisn. water extract improves diabetes and modulates gut microbiota. J Ethnopharmacol 239: 111854.

6. (2014) National Intellectual Property Administration, PRC, CN102824480B: The composition and prepration of a blood glucoselowering auxiliary formula composing of Edgeworthia gardneri.

7. Tong S, Yan J, Chen G, Lou J (2009) Purification of rutin and nicotiflorin from the flowers of Edgeworthia chrysantha Lindl. by high-speed counter-current chromatography. J Chromatogr Sci 47(5): 341-344.

8. Xu P, Xia Z, Lin Y (2012) Chemical constituents from Edgeworthia Gardneri (Thymelaeaceae). Biochem Syst Ecol 45: 148-150.

9. Li XN, Tong SQ, Cheng DP, Li QY, Yan JZ (2014) Coumarins from Edgeworthia chrysantha. Molecules. 19(2): 2042-2048.

10. Nan CY, Zhu JX, Jiang W, Zhong GY, Li M (2017) Chemical constituents from Edgeworthia gardneri Flos. Journal of Chinese Medicinal Materials 40(7): 1618-1621.

11. Zhao DG, Zhou AY, Du Z, Zhang Y, Zhang K, et al. (2015) Coumarins with $\alpha$-glucosidase and $\alpha$ - amylase inhibitory activities from the flower of Edgeworthia Gardneri. Fitoterapia 107: 122-127.

12. Ma YY, Zhao DG, Zhou AY, Zhang Y, Du Z, et al. (2015) $\alpha$-Glucosidase inhibition and antihyperglycemic activity of phenolics from the flowers of Edgeworthia gardneri. J Agric Food Chem. 63(37): 8162-8169.

13. Zhuang M, Qiu H, Li P, Hu L, Wang Y, et al. (2018) Islet protection and amelioration of type 2 diabetes mellitus by treatment with quercetin from the flowers of Edgeworthia gardneri. Drug Des Devel Ther 12: 955966. 
14. Zeng YL, Li L (2008) Optimization on craft and hypoglycemic effect research of SFE-CO2 extractive for Luluohua. Chin J Ethnomed Ethnopharm 10: 7-9.

15. Gao D, Zhang YL, Xu P, Lin YX, Yang FQ, et al. (2015) In vitro evaluation of dual agonists for PPAR $\gamma / \beta$ from the flower of Edgeworthia gardneri (Wall.) Meisn. J Ethnopharmacol 162: 14-19.

16. Chan CM, Chan YW, Lau CH, Lau TW, Lau KM, et al. (2007) Influence of an anti-diabetic foot ulcer formula and its component herbs on tissue and systemic glucose homeostasis. J Ethnopharmacol. 109(1): 10-20.

ISSN: 2574-1241

DOI: 10.26717/BJSTR.2020.28.004717

Chun-Lin Long, Clara Bik-San Lau. Biomed J Sci \& Tech Res

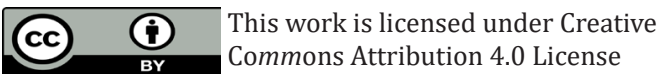

Submission Link: https://biomedres.us/submit-manuscript.php
17. Reagan-Shaw S, Nihal M, Ahmad N (2008) Dose translation from animal to human studies revisited. FASEB J 22(3): 659-661.

18. Wang QY, Xu HY, Xu ZH, Lu ZM, Liu M, et al. (2014) Hypoglycemic effect of water extracts from Edgeworthia gardneri (Wall.) Meisn on type 2 diabetic mice. Nat Prod Res Dev 26: 1385-1388.

\begin{tabular}{ll}
\hline \\
RIOMEDICAL
\end{tabular}$\quad \begin{aligned} & \text { Assets of Publishing with us } \\
& \text { - Global archiving of articles }\end{aligned}$

